This item was submitted to Loughborough's Research Repository by the author.

Items in Figshare are protected by copyright, with all rights reserved, unless otherwise indicated.

\title{
Using the think aloud protocol to measure desire-goal conflict and conflict resolution in a postural persistence task
}

PLEASE CITE THE PUBLISHED VERSION

https://doi.org/10.1080/1091367x.2020.1835663

\section{PUBLISHER}

Informa UK Limited, trading as Taylor \& Francis Group

\section{VERSION}

AM (Accepted Manuscript)

\section{PUBLISHER STATEMENT}

This is an Accepted Manuscript of an article published by Taylor \& Francis in Measurement in Physical Education and Exercise Science on 2 Nov 2020, available online:

http://www.tandfonline.com/10.1080/1091367X.2020.1835663.

\section{LICENCE}

CC BY-NC-ND 4.0

\section{REPOSITORY RECORD}

Gunn, Christopher, and lan Taylor. 2020. "Using the Think Aloud Protocol to Measure Desire-goal Conflict and Conflict Resolution in a Postural Persistence Task". Loughborough University. https://hdl.handle.net/2134/13607627.v1. 
Running head: THINK ALOUD AND DESIRE GOAL CONFLICT

1

2

3

4

5

6

7 Using the Think Aloud Protocol to Measure Desire-Goal Conflict and Conflict Resolution in 8

9

10

11

12

13

14

15

16

17

18

19

20

21

22

23

24

25

a Postural Persistence Task

Manuscript Submitted: March 11th, 2020

Manuscript Resubmitted: July 11th, 2020

Manuscript Resubmitted: September $10^{\text {th }}, 2020$

12

13 


\section{Abstract}

27

The aim of the present study was to investigate a think-aloud protocol as a novel way to measure indicators of self-control during acts of physical persistence. Using a within-person design, 35 participants ( 9 male, 26 female) completed two wall-sit persistence tasks, one employing a think aloud protocol, and the other silent. A two-way mixed ANOVA found no significant differences in persistence between conditions, indicating that using the think aloud protocol during the wall-sit did not have a significant effect on persistence. Think aloud scripts of 34 participants (one participant's script was inadmissible) revealed that all participants verbalized negative affect, an essential characteristic of a desire-goal conflict. Thirty-two participants (94 percent) verbalized conflict resolution strategies. A multiple linear regression found that the length of time from the beginning of the task to the first verbalization of negative affect positively predicted persistence on the wall-sit task $(\beta=.61 ; p$ $<0.001)$, but the length of time to the first verbalization of conflict resolution $\operatorname{did} \operatorname{not}(\beta=$ $.16 ; p=.30)$. This indicates that the time at which negative affect is initially verbalized is a reliable predictor of persistence. The study supports the use of the think aloud protocol in postural persistence tests and other simple physical tasks and supports the possibility that verbalization of negative affect may be an indicator of the initiation of a desire-goal conflict.

Key words: Self-control, Think-aloud, Negative-affect, Persistence 
Using the think aloud Protocol to Measure Desire-Goal Conflict and Conflict Resolution in a Postural Persistence task

In many human endeavors, the ability to persist and endure are significant components of success. Endurance athletes, military personnel, the emergency services and individuals in many other fields all encounter a variety of physical and mental discomforts during their activities. In order to achieve their objectives, an ability to withstand or suppress these barriers in favor of goal pursuit is required. These acts of self-control are critical to many life domains, yet this characteristic is poorly understood. The aim of the present study was to investigate a think-aloud protocol as a novel way to measure indicators of self-control during acts of physical persistence.

Greater self-control is related to desirable outcomes across many domains including education (e.g. Duckworth \& Carlson, 2013) and health (Forestier et al., 2018; Schlam et al., 2013). In sport, greater self-control can distinguish professional from recreational athletes (Martin et al., 2016), and is related to superior performance (Cona et al., 2015). Given this significance, understanding how to optimise self-control is critical and can be achieved by identifying and investigating its component parts. Self-control has been defined as 'the capacity to resist a temptation that is in conflict with a desired long-term goal, in order to protect this valued goal' (Fishbach \& Woolley, 2018, pp. 167). This definition highlights three important components: a desire, a higher-order goal and a conflict between the two (Kotabe \& Hoffman, 2015). In endurance sport, a higher order goal might be optimal performance, winning a race or simply exerting maximal subjective effort. Conversely, a desire exists to slow down, disengage from the activity or reduce effort in order to relieve the different facets of discomfort typical of endurance acts. These two components continuously vary in strength and degree of conflict. 
Self-control can be separated into two distinct phases: the identification of a desiregoal conflict and subsequent conflict resolution strategies to mitigate the conflict (Fishbach \& Woolley, 2018). When the desire and higher order goal are incompatible, the desire becomes a temptation and barrier blocking achievement of the higher order goal. An endurance runner, for example, may begin to evaluate feelings of fatigue as being excessive and a desire to relieve the feelings comes in conflict with the performance goal. Following conflict identification, conflict resolution is sought through the inhibition of responses or modification of behavior. These latter resolution strategies are typically evident and can be quantified experimentally, such as reaction times during stop-signal tasks (Congdon et al., 2012) or when a continuous handgrip falls below a particular force (Bray et al., 2008). Resolution strategies are also easily observed in real-world contexts, such as a deep breath when attempting to stop an aggressive reaction or a grimace when fighting discomfort during endurance performance. However, what is less apparent is when a desire-goal conflict is identified. Often, this stage is measured using neuroimaging of anterior cingulate cortex activation (Botvinick, et al., 2001) or error-related negativity (Legault \& Inzlicht, 2013), which represent practical and measurement issues during physical endurance tasks. Being able to measure the identification of desire-goal conflict using more overt techniques is important as forestalling this moment may be a promising avenue to enhance performance (Taylor et al., 2018).

The identification of a desire-goal conflict is a conscious process (Fishbach \& Woolley, 2018) and goal-related costs and benefits are cognitively highly accessible during endurance tasks (Brandstatter \& Schuler, 2013). Therefore, it may be possible to verbalize such episodes. Indeed, verbal reports have been used previously to gain insight into cognitive conflicts when choosing whether to continue or to disengage from goal pursuit (e.g., Buman, et al., 2008). Typically, however, retrospective reports of experiences are used, which might 
be inaccurate due to temporal bias (Nisbett \& Wilson, 1977). Therefore, a useful approach is concurrent verbalization, in which individuals verbalize their thoughts during a task (Ericsson \& Simon, 1993). Information expressed instantaneously is processed in short-term memory, therefore, it is thought to represent a more accurate reflection of one's thoughts and cognitions, compared to retrospective accounts.

'Think aloud' protocols have been used in athletic populations (Hoffmann \& Loughead, 2019), including collecting thought processes in endurance sports such as running (Samson at al., 2017) and cycling (Whitehead et al., 2018). It has been argued that using a think aloud protocol can disrupt cognitive processes and affect performance (Wilson, 1994). However, if only task relevant thoughts (i.e., Level 1 verbalizations), or visual images or abstract concepts that have been recoded into words (Level 2 verbalizations) are expressed, it is has been proposed that performance will not be affected. Only verbalizations regarding reactive explanations, justifications and reasoning (Level 3) are detrimental to performance (Fox, et al., 2011). This debate has mainly centred around problem solving, memory and decision-making tasks, rather than endurance activities. Potentially positive effects of think aloud protocols exist, such as increased focus and concentration, as well as negative effects including conserving energy from the task at hand in order to think aloud (Whitehead et al., 2018). However, the direct impact on performance is not clear. The first objective of the study, therefore, was to determine if using a think aloud protocol influenced persistence on a physical task that required resisting a desire to quit in favor of a performance goal.

Clarification of this issue is significant to enable future work to use this method to develop understanding of self-control.

Think aloud protocols may facilitate the investigation of desire-goal conflicts and subsequent resolution strategies through the content of participants' verbalizations. Most notably, the onset of the desire-goal conflict is characterized by negative affective states 
124 (Kurzban et al., 2013). The primary role of negative affect is to motivate action because there

is a discrepancy between desired and current state (Carver, 2006). This negative affect can be underpinned by the visceral sensations of a departure from homeostasis (Craig, 2013) or the desire-goal conflict itself (Inzlicht \& Legault, 2014). Either way, negative affect exists to signal the desire-goal conflict and this state is adaptive, orienting people to the conflict as well as motivating them to overcome or manage the conflict (Legault \& Inzlicht, 2013). As such, expressions indicative of typical negative affective states, such as distress (Watson et al., 1988), may be reliable indicators of desire-goal conflict identification. Verbal indicators of subsequent strategies designed to dampen down urges or inhibit response tendencies may indicate subsequent conflict resolution processes. Based on this idea, the second objective of the study was to examine whether participants frequently and reliably verbalized indicators of desire-goal conflict initiation (i.e., negative affect) or subsequent conflict resolution strategies. Because these two phases of self-control may be important for physical persistence, we also investigated whether the length of time from the beginning of the task to first verbalization of negative affect and conflict resolution predicted persistence. Doing so will facilitate understanding of what and when self-control processes occur during persistence tasks and represents an important step in advancing the measurement of desire-goal conflicts during performance.

In the present study, it was expected that task-relevant thoughts, rather than reactive justifications, would be verbalized by participants (Fox et al., 2011). Hence, it was hypothesized that a think-aloud protocol would not influence persistence on a postural endurance task. Our aim of identifying reliable verbal indicators of desire-goal conflict initiation and resolution strategies was more exploratory. Nonetheless, based on theories of self-control we hypothesized that participants would verbalize negative affective states and conflict resolution strategies. We aimed to identify the percentage of participants verbalizing 
these aspects and when during the persistence task they first did so. To investigate the importance of negative affect as an indicator of conflict initiation in predicting persistence, we hypothesized that the length of time from the beginning of the task to when negative affective states were first indicated would be positively associated with persistence on an endurance task (i.e., earlier indication of negative affect would be associated with reduced persistence). We also examined the relationship between first verbalization of conflict resolution and task persistence, although we were cognizant of contrasting perspectives.

While effective use of conflict resolution strategies has been associated with greater persistence (e.g. Wolff et al., 2018), using such strategies may deplete available resources, leading to lower persistence (e.g., Baumeister \& Vohs, 2003).

\section{Method}

\section{Participants}

A study advert was placed on an online university research participation scheme forum along with other studies for students to choose which studies they wished to participate in for course credits. The obtained sample consisted of 35 participants ( 9 male, 26 female) aged 18-35 years old ( $M$ age $=20$ years; $S D=3$ years), all of which completed both conditions. No existing research could assist in power calculations, however, for the mixed ANOVA examining differences in persistence across experimental conditions, the sample size was sufficient to detect an interaction (between order and experimental effects) effect size $(f)$ of $.13(1-\beta=.85, \alpha=.05$, correlation among repeated measures $=.88)$.

\section{Procedures}

Following approval from a university ethics committee, each participant provided informed consent after the procedure had been explained and it was clarified that involvement was anonymous and voluntary. All participants were healthy, as assessed by a university approved general health questionnaire. 

a wall-sit persistence task. Prior to the wall-sit task, participants completed questionnaires to allow for the assessment of differences in physical fatigue and exhaustion in both experimental conditions. Participants were then familiarized with the wall-sit procedure. Specific exercise instructions were scripted so that they remained constant for each participant. Participants were directed to stand with their backs against a wall, feet shoulder width apart and knees and hips flexed at a 90-degree angle, with hands resting against the wall. The aim of the task was to stay in that position for as long as possible. Hence, participants needed to resist a proximal and increasing desire to stop due to increasing discomfort in favor of the distal performance goal of maintaining the position for as long as possible (i.e., a desire-goal conflict). The wall-sit has been successfully used in previous studies of self-control (e.g., Boat \& Taylor, 2017) and is physically challenging yet provides adequate opportunity for participants to verbalize their thoughts.

This procedure was identical in both conditions except, in one condition, participants were asked to perform the wall sit procedure while thinking aloud and in the other condition they were silent (i.e., control condition). Conditions were counterbalanced so that participants were assigned to either a think aloud or control condition first. Each participant performed the task alone, and experimental conditions were seven days apart. In the think aloud condition, the procedure was consistent with Ericsson and Simon's (1980) recommendations for a think aloud protocol, in which individuals verbalize their thoughts concurrently while doing a task. Participants were given a brief introduction to the think aloud protocol, then practiced think aloud exercises, which consisted of naming the fourth letter after the letter L in the alphabet, multiplying 19 and six, and naming 20 animals. These tasks allowed participants to become familiar with the think aloud procedure and allowed the experimenter to gauge if they needed further practice or not. For example, if participants did not think 
aloud while finding the fourth letter after ' $\mathrm{L}$ ', the experimenter demonstrated himself with a different letter.

Participants were instructed to think aloud during the wall-sit task and a) focus on feelings and thoughts; b) not to explain thoughts; c) not to speak to the experimenter and imagine that they are alone; and d) not to worry about disjointed thoughts or the kind of language used. These instructions were designed with Ericson and Simon's (1980) guidelines in mind to avoid the augmenting of verbalizations with descriptions or explanations. To further ensure this, the experimenter was out of the participant's sight but could see the participant through a one-way mirror. The original guidelines suggest the use of verbal probes to encourage participants to think aloud, However, they were used only when absolutely necessary to avoid overly influencing participants' thought processes (Dietrich \& Ehrlenspiel, 2010). All think aloud instructions were scripted to ensure uniformity of instruction. All audio recordings were transcribed verbatim, with statements grouped into sub themes and then major themes.

\section{Measures}

\section{Fatigue and Exhaustion}

Two items were used from the Profile of Mood States Questionnaire (POMS) (McNair et al., 1981) to measure pre-task fatigue and exhaustion in both experimental conditions. Participants were instructed to identify how they were currently feeling for each item on a 5-point scale from 1 (not at all) to 5 (extremely). These items have been employed in previous self-control research (Boat \& Taylor, 2017) and the POMS has established reliability and validity (Beedie et al., 2000). The two items chosen (fatigued and exhausted) were chosen due to being associated with the highest factor loadings on the fatigue subscale (.64 and .66 respectively).

\section{Desire Goal Conflict Identification and Conflict Resolution Strategies}



the start of the wall-sit until the first verbalization of negative affect, which represents an essential feature of the conflict. Potential verbalizations included negative affective states

227

(e.g., distress, frustration) or expressions of pain or discomfort. Initiation of conflict resolution strategies was measured by the time (in seconds) from the start of the wall-sit until the first verbalization of a conflict resolution strategy, such as self-encouragement or distractions.

\section{Persistence}

Participants' persistence was measured as the total time in seconds that they maintained the wall-sit position.

\section{Preliminary Analysis}

Means and standard deviations for pre-task exhaustion and fatigue, persistence in wall-sit task, and the time of first statements referring to negative affect and conflict resolution can be seen in Table 1. Shapiro-Wilk's tests indicated that the scores for fatigue and exhaustion were not normally distributed $(W s=.73-.82$; all $p<.001)$. A number of methods were attempted to normalize the data, including log, square root and reciprocal transformations, however, none were successful. Hence, two non-parametric Wilcoxon signed rank tests were performed to determine if there were differences in fatigue and exhaustion across silent and think aloud conditions. There was no significant difference in fatigue in the silent compared to the think aloud condition $(r=.21 ; z=1.79, p=.07)$. A second Wilcoxon signed-rank test determined that there was a no significant difference in exhaustion in the silent condition compared to the think aloud condition $(r=.14 ; z=1.16, p=$ $.25)$.

\section{Does using think aloud influence persistence?}


(think-aloud protocol completed first or second) and experimental condition as the withinperson factor was employed to determine if there were differences in persistence in the wall-

252 sit. Shapiro-Wilk's tests indicated that the data were not normally distributed ( $W s=.89-.91$; all $p<.01$ ), however, a log transformation was applied and a subsequent Shapiro-Wilk's tests indicated data were normally distributed $(W s=.95-.98 ; p>.36)$. Homogeneity of variances (Levene's Test $F=(1,33) .54, .91 ; p \geq .61)$ and covariances (Box's test: $F=.39 ; p=.76)$ were established.

Using the log of the data, main effects of experimental condition and order effects were not statistically significant and effect sizes were trivial. There was also no statistically significant interaction between experimental conditions and the order they took part in each condition with a trivial effect size (see table 2). To aid interpretation, back-transformed mean scores for persistence in each experimental condition are shown in Table 1 . These results indicate that using the think aloud protocol during the wall-sit did not have a significant effect on persistence.

\section{Frequency across participants of verbal accounts of negative affect and conflict} resolution.

One script was not analyzed because the participant spoke entirely in Hungarian, leaving 34 scripts in total.

Negative affect. Thirty-four participants (100 percent) verbalized negative affect. These verbalizations sometimes described the feeling of the posture itself (e.g., 'it's a bit of an uncomfortable position') but often explicitly described the pain participants were trying to resist ('it's really burning my legs' or 'my knees are dying'). Participants also expressed negative affect such as 'this is embarrassing' and 'they keep hurting more, that's annoying'. 
Conflict resolution. Thirty-two participants (94 percent) verbalized some form of

conflict resolution. These verbalizations took the form of self-encouragement, such as 'keep going' or 'stay with it, come on', as well as instructing themselves about correct technique including posture and positioning (e.g., 'I find it easier if I look down'), and breathing patterns (e.g., 'need to remember to breathe'). Participants also verbalized techniques to distract themselves from the discomfort of the task. These abstract verbalizations came in the form of internal dissociation or inward distractions (e.g., 'cat, 'lions', 'what am I going to do next?'), as well as simply counting numbers.

\section{Are first verbalizations of negative affect and conflict resolution associated with}

\section{persistence?}

A multiple linear regression was conducted to predict persistence from first verbalization of negative affect and first verbalization of conflict resolution. The multiple regression model statistically significantly predicted persistence $F(2,29)=9.06, p=0.001$ ). Time until first verbalization of negative affect significantly predicted persistence $(\beta=.61 ; p$ $<0.001)$, but time until first statement of conflict resolution $\operatorname{did}$ not $(\beta=.16 ; p=.30)$. Adjusted $R^{2}$ for the overall model was $34.2 \%$, a large size effect according to Cohen (1988). See table 3 .

Applying a Bonferroni correction to account for four tests (two Wilcoxon signed rank tests, ANOVA, linear regression; $p=.0125$ ) would not change any of the conclusions presented based on traditional significance criteria $(p=.05)$.

\section{Discussion}

The first objective of the study was to determine whether the think aloud protocol would influence persistence on a postural task. This was important because it helps clarify the suitability of the method for future investigation into self-control. The small effect size (partial $\eta^{2}=.01$ ) of experimental effects on persistence support the idea that employing a 
think aloud protocol to measure desire-goal conflict and conflict resolution strategies does not significantly influence persistence on a postural maintenance task. This implies that, as long as participants verbalize task relevant thoughts that they have recoded into words (Level 1 and 2 verbalizations) and are primed to avoid explanations and justifications (Level 3), the think aloud protocol will not have an impact on persistence (Fox et al., 2011). Participants were not positively influenced, resulting in increased focus and concentration, nor negatively impacted by transferring energy from the task itself to think aloud. These findings are novel because they examine the impact of think-aloud methods on persistence in physical rather than mental tasks. As a result, researchers can have confidence in using this method to investigate self-control processes during similar endurance or persistence tasks. Moreover, the finding that persistence was not influenced by asking participants to verbalize thoughts enables the use of the think aloud protocol to examine other cognitive phenomena during persistence tasks, such as attentional focus.

In addition to the lack of effect on persistence, the content of the verbalizations potentially provides knowledge concerning the desire-goal conflict. All participants verbalized negative affective states during the persistence task. This was expected because the onset of the desire-goal conflict is characterized by negative affective states (Kurzban et. al., 2013). The frequency of these expressions (i.e.,100 percent of the sample) is important because it would not be practical to employ this method if significantly fewer participants expressed negative affect. Importantly, the first indication of these negative affective states significantly predicted persistence and the effect size indicated that this relationship was substantial. That is, the earlier participants started to experience negative affect, the less they persisted. This relationship was important to establish because it indicates that the later a participant begins to experience (or at least verbalize) negative affect, the later they are likely to succumb to the desire to quit. The fact that negative affect significantly predicts 
persistence implies it is a marker of desire-goal conflict identification. Of course, it is plausible that the negative affect could signal something other than a desire-goal conflict. However, the desire-goal conflict is a highly accessible conscious process during physically effortful activity (Brandstatter \& Schuler, 2013) and psychophysiological sensations underpinning the desire to disengage tend to dominate attention during acts of endurance (Hutchinson \& Tenenbaum, 2007). In a wall-sit task there is little tactical or technical thought required, nor is boredom likely in a relatively brief task. As a result, we think it is reasonable to assume that the negative affect represents an acceptable indicator of desire-goal conflict initiation.

Investigation of the desire-goal conflict represents a step forward as much of the selfcontrol research has focused on failures in self-control (i.e., the end of self-control, rather than the beginning), such as a handgrip falling below a particular force (Bray et al., 2008). Here, we demonstrate that the initiation of self-control, in the form of a negative affect marker of the identification of a desire-goal conflict, may also be important. This supports the multicomponent view of self-control (Kotabe \& Hoffmann, 2015), in which different stages of self-control have unique implications for performance. Specifically, the findings imply that forestalling the initiation of a desire-goal conflict might enhance endurance (Taylor et al., 2018).

In addition to the expression of negative affect, 32 participants verbalized some form of desire-goal conflict resolution, which aligns with the two phases of self-control (e.g., Fishbach \& Woolley, 2018). Although not all of the sample expressed conflict resolution strategies, 94 percent is still a practically useful number of participants. Much of these expressions could be classified as associative (monitoring) or dissociative (distractions; Brick et al, 2014; Stevinson \& Biddle, 1998). Examples of internal associative thoughts were those related to breathing patterns or correct posture and technique. Externally associative 
strategies often consisted of counting to ten or breaking the task down into smaller short-term goals (Brick et al., 2015). Dissociative thoughts were mainly internally directed, such as plans for later in the day and more abstract thoughts. Involuntary or external distractions, such as the scenery or other participants, were limited due to the mundane laboratory setting used in the present study.

Despite the frequency of conflict resolution verbalizations, the time of first verbalization did not significantly predict persistence on the task and the effect size was small, suggesting limited association. In other words, early use of conflict resolution strategies did not lead to better performance. Self-control is undoubtedly important in the sports domain, particularly so for endurance activities in which increasing discomfort and the desire to reduce effort must be overcome. In line with this assumption, conflict resolution strategies, such as self-talk (Hamilton et al., 2007), goal setting, imagery and relaxation (Arthur et al., 2017) have been associated with enhanced endurance performance. The present study, albeit tentatively, suggests an alternative means through which to improve self-control, in which the focus of such interventions could be put on delaying the onset of negative affect and discomfort, rather than persisting through it.

\section{Limitations and Future Directions}

While the findings in the present study suggest the suitability of the think aloud protocol to measure self-control processes in a postural persistence test, the task used in the present study required little motor skill and was largely unfamiliar to the participants.

Therefore, asking participants to think aloud might have different implications in well trained individuals or in more complex settings such as endurance events, in which the nature of verbalizations might be different. While the think aloud protocol has been validated in running (Samson et al., 2017) and cycling (Whitehead et al., 2018) environmental factors could have implications on the frequency, timing and nature of verbalizations of desire-goal 
conflict or conflict resolution strategies. Therefore, use of the think aloud protocol to measure self-control processes should be tested in other more complex domains to confirm generalizability.

In comparison to other methods to identify and measure self-control processes, such as neuroimaging of anterior cingulate cortex activation (Botvinick et al., 2001) or using EEG signals to measure error-related negativity (Legault \& Inzlicht, 2013), the think aloud protocol represents few practical issues, especially when used in the context of physical persistence tasks. The motivational value of desires and goals during endurance has been measured using self-evaluations during cycling trials (Taylor et al., 2020) but this method does not provide information on the initiation of the desire-goal conflict and associated negative affect. Despite the use of familiarization activities, there is a likelihood that not all thoughts and feelings would have been accurately verbalized, particularly those that are abstract in nature (Level 2 verbalizations; Fox et al., 2011). Therefore, further testing, possibly alongside the above-mentioned methods could provide a clearer picture as to the reliability of the think aloud method in measuring self-control processes.

As was alluded to earlier, the primary function of negative affect is to motivate remedial action towards a desired state (Carver, 2006), therefore, negative affect can be reasonably attributed as an indicator of a desire-goal conflict. However, it cannot be ruled out that negative affect is a marker of some other phenomena distinct from the initiation of a desire-goal conflict. Hence, future research should seek to clarify the underpinning reason for negative affective states.

As we speculate in our evaluation of the study data, future research could focus on mechanisms through which the onset of the desire-goal conflict (and associated negative affect) could be delayed. The psychology of means-ends fusion (Kruglanski et al., 2018) describes a perceptual fusion of an activity and its goal, with the degree to which they are 
398 fused reflecting how intrinsically motivating the activity is to an individual. Therefore, it 399 could be of great value to fuse the goal of optimal performance with the activity of persisting 400 through discomfort. Hence, rather than seeing physical discomfort as conflicting with the 401 goal, it could be seen as in line with the goal. With a high degree of fusion, the positive 402 motivational and affective properties of optimal performance could be transferred from the 403 goal to the activity of resisting discomfort, delaying the onset of negative affect and

404 improving persistence.

405

\section{Conclusion}

In conclusion, this study supports the use of the think aloud protocol in postural persistence tests and other simple physical tasks. Further to this, verbalizations of negative affect may be a reliable indicator of the initiation of the desire-goal conflict, which suggests they can be used effectively in the study of self-control. 
Arthur, R. A., Fitzwater, J., Roberts, R., Hardy, J., \& Arthur, C. A. (2017). Psychological skills and "the Paras": The indirect effects of psychological skills on endurance. Journal of Applied Sport Psychology, 29(4), 449-465. https://doi.org/10.1080/10413200.2017.1306728

Baumeister, R. F., \& Vohs, K. D. (2003). Self-regulation and the executive function of the self. Handbook of Self and Identity, 1, 197-217.

Beedie, C. J., Terry, P. C., \& Lane, A. M. (2000). The profile of mood states and athletic performance: Two meta-analyses. Journal of Applied Sport Psychology, 12, 49-68. https://doi.org/10.1080/10413200008404213

Boat, R., \& Taylor, I. M. (2017). Prior self-control exertion and perceptions of pain during a physically demanding task. Psychology of Sport and Exercise, 33, 1-6. http://doi.org/10.1016/j.psychsport.2017.07.005

Botvinick, M. M., Braver, T. S., Barch, D. M., Carter, C. S., \& Cohen, J. D. (2001). Conflict monitoring and cognitive control. Psychological review, 108(3), 624. https://doi.apa.org/doi/10.1037/0033-295X.108.3.624

Brandstätter, V., \& Schüler, J. (2013). Action crisis and cost-benefit thinking: A cognitive analysis of a goal-disengagement phase. Journal of Experimental Social Psychology, 49(3), 543-553. https://doi.org/10.1016/j.jesp.2012.10.004

Bray, S. R., Martin Ginis, K. A., Hicks, A. L., \& Woodgate, J. (2008). Effects of selfregulatory strength depletion on muscular performance and EMG activation. Psychophysiology, 45(2), 337-343. https://doi.org/10.1123/jsep.35.3.239

Brick, N., MacIntyre, T., \& Campbell, M. (2014). Attentional focus in endurance activity: new paradigms and future directions. International Review of Sport and Exercise Psychology, 7(1), 106-134. https://doi.org/10.1080/1750984X.2014.885554 
Brick, N., MacIntyre, T., \& Campbell, M. (2015). Metacognitive processes in the selfregulation of performance in elite endurance runners. Psychology of Sport and Exercise, 19, 1-9. https://doi.org/10.1016/j.psychsport.2015.02.003

Buman, M. P., Omli, J. W., Giacobbi Jr, P. R., \& Brewer, B. W. (2008). Experiences and coping responses of "hitting the wall" for recreational marathon runners. Journal of Applied Sport Psychology, 20(3), 282-300. https://doi.org/10.1080/10413200802078267

Carver, C. S. (2006). Approach, avoidance, and the self-regulation of affect and action. Motivation and Emotion, 30(2), 105-110. https://doi.org/10.1007/s11031-0069044-7.

Cohen, J. (1988). Statistical power analysis for the behavioral sciences, 2nd ed. Erlbaum.

Cona, G., Cavazzana, A., Paoli, A., Marcolin, G., Grainer, A., \& Bisiacchi, P. S. (2015). It’s a matter of mind! Cognitive functioning predicts the athletic performance in ultramarathon runners. PloS one, 10(7). https://doi.org/10.1371/journal.pone.0132943

Congdon, E., Mumford, J. A., Cohen, J. R., Galvan, A., Canli, T., \& Poldrack, R. A. (2012). Measurement and reliability of response inhibition. Frontiers in psychology, 3, 37. https://doi.org/10.3389/fpsyg.2012.00037

Craig, A. D. (2013). An interoceptive neuroanatomical perspective on feelings, energy, and effort. The Behavioral and brain sciences, 36(6), 685-726. https://doi.org/10.1017/S0140525X13001489

Dietrich, H. \& Ehrlenspiel, F. (2010) Cognitive Interviewing: A Qualitative Tool for Improving Questionnaires in Sport Science. Measurement in Physical Education and Exercise Science, 14(1), 51-60. https://doi.org/10.1080/10913670903455025

Duckworth, A. L., \& Carlson, S. M. (2013). Self-regulation and school success. In B. W. Sokol, F. M. E. Grouzet, \& U. Muller (Eds.), Self-regulation and autonomy: Social 
and developmental dimensions of human conduct (pp. 208-230). Cambridge University Press.

Ericsson, K. A., \& Simon, H. A. (1980). Verbal reports as data. Psychological review, 87(3), 215. https://psycnet.apa.org/doi/10.1037/0033-295X.87.3.215

Ericsson, K. A., \& Simon, H. A. (1993). Protocol analysis: Verbal Reports as data. MIT press.

Fishbach, A.M. \& Woolley, K. (2018). Combatting temptation to promote health and wellbeing. In D. de Riddler, M. Adriaanse, \& K. Fujita (Eds.), Routledge International handbook of self-control in health and well-being (pp. 167-179). Routledge.

Forestier, C., Sarrazin, P., Allenet, B., Gauchet, A., Heuzé, J. P., \& Chalabaev, A. (2018). "Are you in full possession of your capacity?". A mechanistic self-control approach at trait and state levels to predict different health behaviors. Personality and Individual Differences, 134, 214-221. https://doi.org/10.1016/j.paid.2018.05.044

Fox, M. C., Ericsson, K. A., \& Best, R. (2011). Do procedures for verbal reporting of thinking have to be reactive? A meta-analysis and recommendations for best reporting methods. Psychological bulletin, 137(2), 316. https://doi.apa.org/doi/10.1037/a0021663

Hamilton, R. A., Scott, D. \& MacDougall, M. P. (2007) Assessing the effectiveness of selftalk interventions on endurance performance. Journal of Applied Sport Psychology. 19, 226-239 (2007). https://doi.org/10.1080/10413200701230613

Hoffmann, M. D., \& Loughead, T. M. (2019). Preliminary development of a questionnaire to assess peer athlete mentoring functions: The athlete mentoring questionnaire (AMQ). Measurement in Physical Education and Exercise Science, 23(1), 10-25. https://doi.org/10.1080/1091367X.2018.1479708 
498 Hutchinson, J. C., \& Tenenbaum, G. (2007). Attention focus during physical effort: The

499

500

501

502

503

504

505

506

507

508

509

510

511

512

513

514

515

516

517

518

519

520

521

522 mediating role of task intensity. Psychology of Sport and Exercise, 8(2), 233-245. https://psycnet.apa.org/doi/10.1016/j.psychsport.2006.03.006

Inzlicht, M., \& Legault, L. (2014). No pain, no gain: How distress underlies effective selfcontrol (and unites diverse social psychological phenomena). In J. P. Forgas \& E. Harmon-Jones (Eds.), Sydney Symposium of Social Psychology. Motivation and its Regulation: The Control Within (p. 115-132). Psychology Press.

Kotabe, H. P., \& Hoffman, W. (2015) On integrating the components of self-control. Perspectives on Psychological Science, 10(5), 618-638. https://doi.org/10.1177\%2F1745691615593382

Kruglanski, A. W., Fishbach, A., Woolley, K., Belanger, J. J., Chernikova, N., Molinario, E., \& Pierro, A. (2018) A structural model of intrinsic motivation: On the psychology of means-ends fusion. Psychological Review, 125(2), 165-182. https://doi.apa.org/doi/10.1037/rev0000095

Kurzban, R., Duckworth, A., Kable, J. W., \& Myers, J. (2013). An opportunity cost model of subjective effort and task performance. Behavioral and brain sciences, 36(6), 661679. https://doi.org/10.1017/S0140525X12003196

Legault, L., \& Inzlicht, M. (2013). Self-determination, Self-Regulation, and the Brain: Autonomy Improves Performance by Enhancing Neuroaffective Responsiveness to Self-Regulation Failure. Journal of Personality and Social Psychology 105(1), 125138. https://doi.apa.org/doi/10.1037/a0030426

Martin, K., Staiano, W., Menaspà, P., Hennessey, T., Marcora, S., Keegan, R., Thompson, K. G., Martin, D., Halson, S. \& Rattray, B. (2016). Superior inhibitory control and resistance to mental fatigue in professional road cyclists. PloS one, 11(7). https://doi.org/10.1371/journal.pone.0159907 
McNair, D. M., Lorr, M., \& Droppelman, L. F. (1981). Edits manual: Profile of Mood States. Educational and Industrial Testing Service.

Nisbett, R. E., \& Wilson, T. D. (1977). Telling more than we can know: Verbal reports on mental processes. Psychological review, 84(3), 231. https://psycnet.apa.org/doi/10.1037/0033-295X.84.3.231

Samson, A., Simpson, D., Kamphoff, C., \& Langlier, A. (2017). Think aloud: An examination of distance runners' thought processes. International Journal of Sport and Exercise Psychology, 15(2), 176-189. https://doi.org/10.1080/1612197X.2015.1069877

Schlam, T. R., Wilson, N. L., Shoda, Y., Mischel, W., \& Ayduk, O. (2013). Preschoolers' delay of gratification predicts their body mass 30 years later. The Journal of pediatrics, 162(1), 90-93. https://doi.org/10.1016/j.jpeds.2012.06.049

Stevinson, C. D., \& Biddle, S. J. (1998). Cognitive orientations in marathon running and "hitting the wall". British journal of sports medicine, 32(3), 229-234. http://dx.doi.org/10.1136/bjsm.32.3.229

Taylor, I. M., Boat, R., \& Murphy, S. L. (2018). Integrating theories of self-control and motivation to advance endurance performance. International Review of Sport and Exercise Psychology, 1-20. https://doi.org/10.1080/1750984X.2018.1480050

Taylor, I. M., Smith, K., \& Hunte, R. (2020). Motivational processes during physical endurance tasks. Scandinavian Journal of Medicine \& Science in Sports. https://doi.org/10.1111/sms.13739

Watson, D., Clark, L. A., \& Tellegen, A. (1988). Development and validation of brief measures of positive and negative affect: the PANAS scales. Journal of personality and social psychology, 54(6), 1063. https://psycnet.apa.org/doi/10.1037/0022$\underline{3514.54 .6 .1063}$ 
548

549

550

551

552

553

554

555

556

557

558

559

560

561

562

563

564

565

566

567

568

569

570

571

572

573

Whitehead, A. E., Jones, H. S., Williams, E. L., Rowley, C., Quayle, L., Marchant, D., \& Polman, R. C. (2018). Investigating the relationship between cognitions, pacing strategies and performance in $16.1 \mathrm{~km}$ cycling time trials using a think aloud protocol. Psychology of Sport and Exercise, 34, 95-109. https://doi.org/10.1016/j.psychsport.2017.10.001

Wilson, T. D. (1994). The Proper Protocol: Validity and Completeness of Verbal Reports. Psychological Science, 5(5), 249-252. https://doi.org/10.1111\%2Fj.1467$\underline{9280.1994 . t b 00621 . x}$

Wolff, W., Bieleke, M., Hirsch, A., Wienbruch, C., Gollwitzer, P. M., \& Schüler, J. (2018). Increase in prefrontal cortex oxygenation during static muscular endurance performance is modulated by self-regulation strategies. Scientific reports, $8(1), 1-10$.

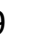

\section{0}

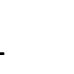

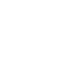


574

575

\begin{tabular}{lcccc}
\hline Variable & \multicolumn{2}{c}{$\begin{array}{c}\text { Think aloud } \\
\text { condition }\end{array}$} & \multicolumn{2}{c}{$\begin{array}{c}\text { Control (silent) } \\
\text { condition }\end{array}$} \\
\hline & $M$ & $S D$ & $M$ & $S D$ \\
\hline Fatigue & 2.43 & 0.92 & 2.14 & 0.98 \\
Exhaustion & 1.8 & 1.05 & 1.66 & 0.91 \\
$\begin{array}{l}\text { Persistence (seconds) } \\
\begin{array}{l}\text { Back-transformed } \\
\text { persistence (control first) }\end{array}\end{array}$ & 124.17 & 65.22 & 122.09 & 67.52 \\
$\begin{array}{l}\text { Back-transformed } \\
\text { persistence (think aloud }\end{array}$ & 116.00 & 65.85 & 111.35 & 65.25 \\
$\begin{array}{l}\text { first) } \\
\begin{array}{l}\text { First statement of negative } \\
\text { affect (seconds) }\end{array}\end{array}$ & 20.03 & 23.73 & & \\
$\begin{array}{l}\text { First statement of conflict } \\
\text { resolution (seconds) }\end{array}$ & 29.63 & 20.39 & & \\
\hline
\end{tabular}

Table 1

Means and Standard Deviations of Study Variables

577

578

579

580

581

582

583

584

585

586

587

588

589 
Table 2

591

Two-Way Mixed ANOVA Examining Differences in Persistence across Order of Conditions

592 as a Between-Person Factor and Experimental Condition as a Within-Person Factor

593

\begin{tabular}{lcclc}
\hline Variable & $F$ & $d f$ & $p$ & Partial eta squared \\
\hline Condition & .29 & 1 & .60 & .01 \\
Order Effects & 1.14 & 1 & .29 & .03 \\
Interaction & .53 & 1 & .47 & .02 \\
\hline
\end{tabular}

594

595

596

597

598

599

600

601

602

603

604

605

606

607

608

609

610

611

612

613

614 
615 Table 3

616 Multiple Regression with Desire-Goal Conflict and Conflict-Resolution as Predictors of 617 Persistence

618

\begin{tabular}{lcccccc}
\hline & $B$ & $S E B$ & $\beta$ & $p$ & $R^{2}$ & $\Delta R^{2}$ \\
\hline Model & 77.20 & 19.41 & & .001 & .39 & .34 \\
D-G Conflict & 1.65 & .39 & .61 & $<.001$ & & \\
Conflict- & .49 & .46 & .16 & .30 & & \\
Resolution & & & & & & \\
\hline
\end{tabular}

619 Note. Desire-Goal (D-G) Conflict = time of first verbalization of desire-goal conflict;

620 Conflict-Resolution $=$ time of first verbalization of conflict resolution strategies.

621

622

623

624

625 